\title{
Comment on "Distribution of Skin-Type Diversity in Photographs in AAD Online Educational Modules"
}

Tejas P. Joshi, BS; Soo Jung Kim, MD, PhD

To the Editor:

We read with great interest the article by $\mathrm{Chu}$ et $\mathrm{al}^{1}$ (Cutis. 2021;107:157-159) and commend them for noting the underrepresentation of skin of color (SOC) in the American Academy of Dermatology (AAD) Basic Dermatology Curriculum. The AAD Basic Dermatology Curriculum represents one introductory resource that is ubiquitously utilized by medical students. Herein, we add an analysis of the representation of SOC in the following resources that also comprise the first exposure medical students have to dermatology: Dermatology Clinics Clinical Advisor articles (https://www.clinicaladvisor.com/home /dermatology/dermatology-clinics/), Learn Derm Module (LDM) by VisualDx (https://www.visualdx. com/learnderm/), Lookingbill and Marks' Principles of Dermatology (6th ed)(LB\&M), ${ }^{2}$ and DermNet NZ (https://dermnetnz.org/). We performed a focused search of the DermNet NZ database for images of the following common dermatologic conditions: acne, rosacea, alopecia, urticaria, arthropod bites, blistering diseases (bullous pemphigoid and pemphigus vulgaris), connective tissue diseases (dermatomyositis and lupus), inflammatory conditions (atopic dermatitis, contact dermatitis, and psoriasis), keloids, benign and malignant neoplasms (nevi, seborrheic keratosis, actinic keratosis, basal and squamous cell carcinomas, and melanoma including acral melanoma), bacterial skin infections (impetigo, erysipelas, cellulitis, staphylococcal scalded skin syndrome, and syphilis), fungal infections (dermatophyte infections), and viral skin infections (herpes, molluscum contagiosum, varicella-zoster virus, and warts). We classified images as light (Fitzpatrick phototypes I-IV) or dark (Fitzpatrick phototypes V or VI). We excluded images without visible background skin (eg, images of oral mucosa, genitalia, nails, palms and soles, dermoscopic images, histopathologic images).

We found the representation of SOC in the resources we selected to be as follows: Dermatology Clinics Clinical Advisor articles (70/367 or 19\%); LDM (26/150 or $17 \%)$; LB\&M (52/374 or $14 \%)$; DermNet NZ (11/310 or $4 \%$ ). Representation of SOC in common dermatologic conditions such as actinic keratosis, alopecia, rosacea, urticaria, and warts was entirely absent across all resources. Other common skin diseases were represented in only one of the resources we analyzed: acne (represented only in LB\&M, where only 3/11 images of acne were depicted in SOC); contact dermatitis (represented only in LB\&M, where only $1 / 6$ images of contact dermatitis were depicted in SOC); psoriasis (represented only on DermNet NZ, where only 2/25 images of psoriasis were depicted in SOC); seborrheic keratosis (represented only in LB\&M, where $1 / 2$ images of seborrheic keratosis were depicted in SOC). Furthermore, none of the resources we analyzed depicted malignancy (basal cell carcinoma, squamous cell carcinoma, and melanoma) in SOC. Although the poor representation of SOC in malignancies can be explained by the predilection of skin cancer for light skin, other dermatologic conditions that are more common in SOC also were poorly represented in these resources in SOC: acral melanoma, not represented in any of the resources we analyzed; subacute cutaneous lupus erythematosus and systemic lupus erythematosus, also not represented in any of the resources we analyzed; keloids, represented only in LB\&M.

From Baylor College of Medicine, Houston, Texas. Mr. Joshi is from the School of Medicine, and Dr. Kim is from the Department of Dermatology. The authors report no conflict of interest.

Correspondence: Tejas P. Joshi, BS, 1 Baylor Plaza, Houston TX 77030 (tejas.joshi@bcm.edu).

doi:10.12788/cutis.0295 
Although no study has investigated the true prevalence of Fitzpatrick phototypes in the United States, He et $\mathrm{al}^{3}$ demonstrated the prevalence of Fitzpatrick phototypes $\mathrm{V}$ and VI to be $25.0 \%$ and $18.8 \%$, respectively, in an ethnically diverse study of 3386 participants. Indeed, the representation of SOC in the resources we analyzed falls short of this plausible estimate of SOC in an increasingly diverse US population.

Our work adds to the growing body of literature exposing the deficiencies in SOC representation in dermatology. As Lester et $\mathrm{al}^{4}$ noted, such poor representation of SOC is deleterious not just to patients, who may be misdiagnosed, but also more generally to the integrity of the field of dermatology. Moreover, our study, which analyzes introductory resources referenced by the junior medical student, highlights a potential danger of poor SOC representation for trainees-limited exposure to SOC may leave medical students unprepared to recognize lesions in SOC during clerkships and residency. Furthermore, we note an additional concern with minimal SOC representation in online modules such as the AAD and LDM module as well as online databases such as DermNet NZ; images from these resources may be used as training sets for machine learning (ML) software (indeed, DermNet NZ has been used as a training set for ML programs ${ }^{5}$ ). However, if data sets with poor representation of SOC are used to train ML algorithms, then ML software may be unable to recognize lesions in SOC.6 Thus, inadequate representation of SOC in online modules and databases may exacerbate existing inequities in dermatology.

To address the paucity of SOC representation, students can be directed to resources devoted to depicting SOC; however, as discussed eloquently by $\mathrm{Chu}$ et $\mathrm{al}^{1}{ }^{1}$ an attempt to update existing resources also must be made. The senior author in our study (S.J.K.) embraced such an approach, updating the dermatology lectures given to medical students to include more images of SOC. Such a top-down approach may represent a major step in dismantling the systemic biases that pervade dermatology.

A limitation of our analysis was use of the Fitzpatrick scale, which was conceived as a phenotypic scale to assess cutaneous responses to UV irradiation. ${ }^{7}$ Although it is the most commonly used scale to describe race/ethnicity and/or constitute skin color, it is not possible to include all non-White skin types and classify strictly under this umbrella term.

\section{REFERENCES}

1. Chu B, Fathy R, Onyekaba G, et al. Distribution of skin-type diversity in photographs in AAD online educational modules. Cutis. 2021;107:157-159. doi:10.12788/cutis.0196

2. Marks JG Jr, Miller JJ. Lookingbill and Marks' Principles of Dermatology. 6th ed. Saunders Elsevier; 2018.

3. He SY, McCulloch CE, Boscardin WJ, et al. Self-reported pigmentary phenotypes and race are significant but incomplete predictors of Fitzpatrick skin phototype in an ethnically diverse population. J Am Acad Dermatol. 2014;71:731-737. doi:10.1016/j.jaad.2014.05.023
4. Lester JC, Taylor SC, Chren M-M. Under-representation of skin of colour in dermatology images: not just an educational issue. $\mathrm{Br} \mathrm{J}$ Dermatol. 2019;180:1521-1522. doi:10.1111/bjd.17608

5. Aggarwal P. Data augmentation in dermatology image recognition using machine learning. Skin Res Technol. 2019;25:815-820. doi:10.1111/srt.12726

6. Adamson AS, Smith A. Machine learning and health care disparities in dermatology. JAMA Dermatol. 2018;154:1247-1248. doi:10.1001 /jamadermatol.2018.2348

7. Ware OR, Dawson JE, Shinohara MM, et al. Racial limitations of Fitzpatrick skin type. Cutis. 2020;105:77-80.

\section{Authors' Response}

We thank Mr. Joshi and Dr. Kim for their reply to our article and their added contribution to the literature on inadequate representation of skin of color (SOC) in dermatology educational materials. In recent years, multiple analyses have reviewed textbooks and popular online resources for SOC representation. ${ }^{1}$ These resources encompass all levels of education-from the laypatient to the medical student, and to residency and beyonddemonstrating the significant challenges to overcome.

In addition, as Mr. Joshi and Dr. Kim state, the potential for these inadequately representative resources to serve as training data for prediction and classification tools adds further urgency to the broader task at hand, as we do not wish to perpetuate disparities. Several tools already exist, including Derm Assist, a recent Googleproduced tool that suggests a list of diagnoses from patient-provided images. ${ }^{2}$ Although Derm Assist has been marked as a CE Class I (low risk) medical device in the European Union, the original research it is built on relied on training data with low representation of darker skin types (2.7\% Fitzpatrick V and 0\% Fitzpatrick VI), ${ }^{3}$ drawing concern for its generalizability.

These concerns about SOC representation are not new; dermatology advocates, scholars, and organizations such as the Skin of Color Society have been working to address these deficiencies for many years, contributing to education (including writing of resources and textbooks) and academic research. This work continues today. For instance, Lester et $\mathrm{al}^{4}$ described best practices for clinical photography in SOC; this guidance was not yet published at the time of our original submission. Not only should dermatology strive for increased quantity of representation but also quality. This metric is particularly important if the images are intended not just for education but also for use as training data for prediction and classification tools.

Examples of more recent actions at the organizational level include the American Academy of Dermatology (AAD) announcing a 3-year plan to promote diversity, equity, and inclusion ${ }^{5}$ and VisualDx establishing \#ProjectIMPACT, a collaboration to reduce health care biases in SOC. ${ }^{6}$ In the AAD 3-year plan, one goal is to "[i] ncrease use of images reflecting full spectrum of skin types and highlight topics on skin of color, health disparities, and cultural competency across all AAD education." ${ }^{5}$ Although

CONTINUED ON PAGE 54 


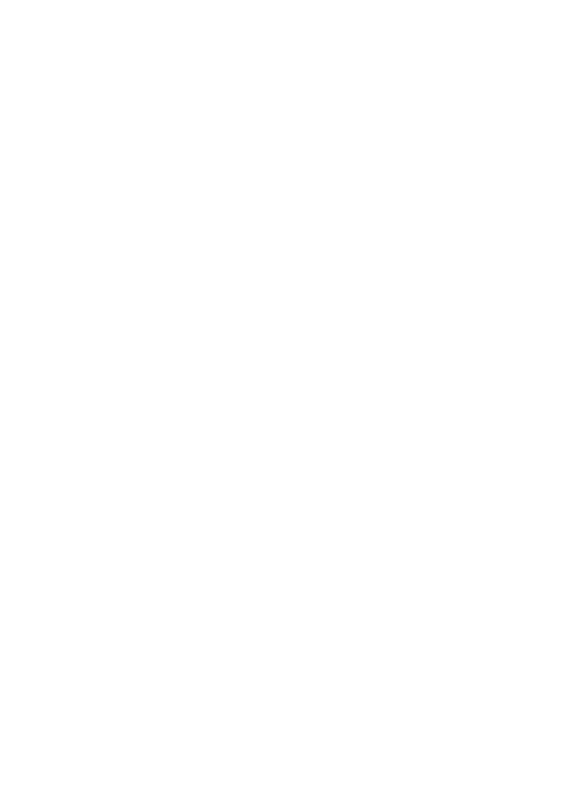

LETTER TO THE EDITOR

CONTINUED FROM PAGE 42

not specifically mentioned, we hope that the AAD has included updating the Basic Dermatology Curriculum, given its inadequate SOC representation, as part of its short-term goals. The greater recognition of these issues through more prevalent analyses published in leading dermatology journals is encouraging, and we hope both that improvements can be successfully implemented and that future studies will reveal improvements in representation.

Brian Chu, BS; Ramie Fathy, AB; Ginikanwa Onyekaba, BS; Jules B. Lipoff, MD

From the Perelman School of Medicine, University of Pennsylvania, Philadelphia. Dr. Lipoff is from the Department of Dermatology and the Leonard Davis Institute of Health Economics.

The authors report no conflict of interest.

Correspondence: Jules B. Lipoff, MD, Department of Dermatology, University of Pennsylvania, Penn Medicine
University City, 3737 Market St, Ste 1100, Philadelphia, PA 19104 (jules.lipoff@pennmedicine.upenn.edu).

\section{REFERENCES}

1. Perlman KL, Williams NM, Egbeto IA, et al. Skin of color lacks representation in medical student resources: a cross-sectional study. Int $J$ Womens Dermatol. 2021;7:195-196. doi:10.1016/j.jiwd.2020.12.018

2. Bui P, Liu Y. Using AI to help find answers to common skin conditions. Published May 18, 2021. Accessed June 12, 2021. https://blog.google /technology/health/ai-dermatology-preview-io-2021

3. Liu Y, Jain A, Eng C, et al. A deep learning system for differential diagnosis of skin diseases. Nature Medicine. 2020;26:900-908. doi:10.1038/s41591-020-0842-3

4. Lester JC, Clark L, Linos E, et al. Clinical photography in skin of colour: tips and best practices. Br J Dermatol. 2021;184:1177-1179. doi:10.1111/ bjd.19811

5. American Academy of Dermatology Association. Diversity in dermatology: diversity committee approved plan 2021-2023. Published January 26, 2021. Accessed June 24, 2021. https://assets.ctfassets .net/1ny4yoiyrqia/xQgnCE6ji5skUlcZQHS2b/65f0a9072811e11afcc33d 043e02cd4d/DEI_Plan.pdf

6. VisualDx. \#ProjectIMPACT. Accessed June 24, 2021. https://www .visualdx.com/projectimpact/ 